PALEO

Revue d'archéologie préhistorique

13 | 2001

Varia

\title{
Nouvelles figures féminines schématiques de type Lalinde-Gönnersdorf dans la vallée de l'Aveyron
}

New schematic female figures (type Lalinde-Gönnersdorf) in the Aveyron valley

\section{Edmée Ladier}

\section{(2) OpenEdition}

Journals

Édition électronique

URL : http://journals.openedition.org/paleo/1114

DOI : $10.4000 /$ paleo.1114

ISSN : 2101-0420

Éditeur

SAMRA

\section{Édition imprimée}

Date de publication : 14 décembre 2001

Pagination : 265-274

ISSN : $1145-3370$

\section{Référence électronique}

Edmée Ladier, « Nouvelles figures féminines schématiques de type Lalinde-Gönnersdorf dans la vallée de l'Aveyron », PALEO [En ligne], 13 | 2001, mis en ligne le 26 mai 2010, consulté le 07 juillet 2020. URL http://journals.openedition.org/paleo/1114; DOI : https://doi.org/10.4000/paleo.1114

Ce document a été généré automatiquement le 7 juillet 2020.

\section{(c) $(1) \odot$}

PALEO est mis à disposition selon les termes de la licence Creative Commons Attribution - Pas d'Utilisation Commerciale - Pas de Modification 4.0 International. 


\title{
Nouvelles figures féminines
} schématiques de type LalindeGönnersdorf dans la vallée de l'Aveyron

New schematic female figures (type Lalinde-Gönnersdorf) in the Aveyron valley

\author{
Edmée Ladier
}

\section{Situation géographique}

1 Le site de La Magdeleine-La Plaine se trouve sur la rive droite de l'Aveyron à 2,5 km environ à vol d'oiseau en aval du village de Penne (Tarn) et à 3,5 km environ en amont de Bruniquel (Tarn-et-Garonne). Il se situe dans les environs immédiats du confluent de l'Aveyron avec un petit ruisseau temporaire, alimenté par une source de faible débit. L'étroite vallée creusée par ce ruisseau constitue un des rares accès au plateau situé en contre-haut. La vallée de l'Aveyron est ici très étroite et très encaissée, bordée en son sommet d'abrupts calcaires parfois surplombants. Le plateau culmine à $307 \mathrm{~m}$ tandis que l'étiage de l'Aveyron est à $101 \mathrm{~m}$.

\section{Situation topographique}

2 Le gisement se trouve à proximité immédiate d'un réseau de grottes se développant sur quatre niveaux et dont les porches ne sont pas exactement superposés.

3 La grotte occupant le deuxième niveau de ce réseau n'est autre que la grotte de la Magdeleine-des-Albis, connue pour les bas-reliefs paléolithiques (Magdalénien moyen probable, Rouzaud et alii 1989) qui ornent ses parois: c'est la grotte des Vénus, découvertes en 1952 par Henri Bessac (Bessac 1971) et authentifiées la même année par l'abbé Breuil (Breuil 1952). Le gisement se trouve à trois mètres environ en contrebas 
de la cavité ornée; il occupe une terrasse d'une douzaine de mètres de large, couverte d'une épaisse végétation de buis, chênes et érables. Ce replat surplombe de 2,5 m environ un chemin de terre qui longe la rive droite de l'Aveyron. Il bénéficie d'une excellente exposition au sud et son altitude par rapport à l'Aveyron, bien que modérée (une douzaine de mètres), le met à l'abri des plus hautes crues actuelles (Carte 1).

Carte 1 : La Magdeleine-La Plaine : situation géographique.

Map 1 : La Magdeleine-La Plaine : geographical location.

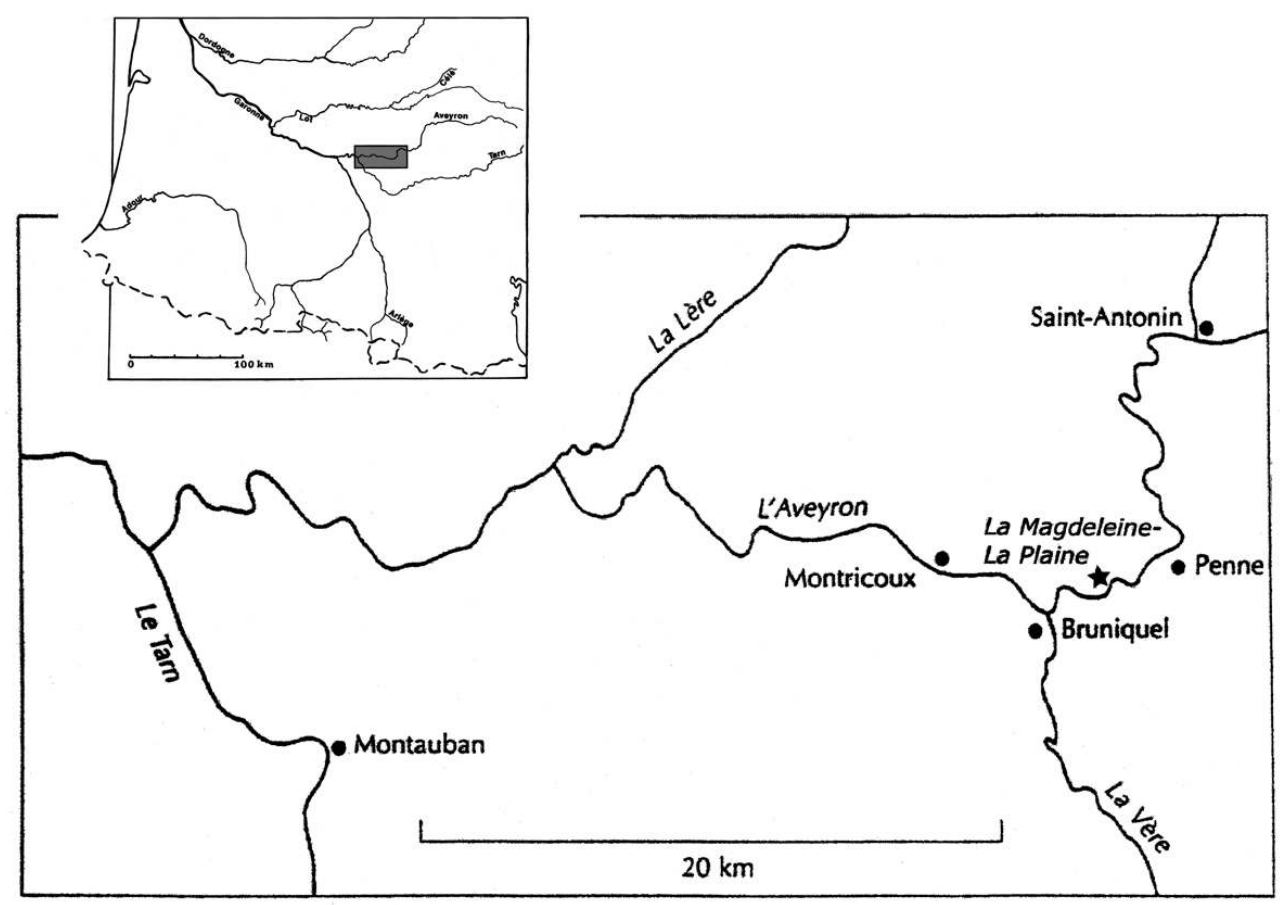

\section{Historique des recherches et travaux antérieurs}

La présence probable d'un gisement paléolithique a été signalée en 1977 par H. Bessac, inventeur des bas-reliefs de la grotte ornée. Des travaux d'élargissement du chemin, entamant le talus, avaient mis au jour en 1971 une abondante industrie lithique ainsi que de la faune fragmentée riche en renne (Bessac 1977).

Par la suite, probablement entre 1976 et 1980, H. Bessac avait pratiqué un petit sondage sur le replat, opération qu'il n'avait jamais publiée mais qui lui avait donné un intéressant mobilier.

6 Le gisement était donc peu et mal connu. Seule la publication de 1977 faisait état du matériel mis au jour lors de l'élargissement du chemin. Le matériel publié par $\mathrm{H}$. Bessac était intéressant par son abondance (plus de 10000 artefacts) et son attribution culturelle (Magdalénien final), corroborée par une radiodatation effectuée à Lyon (Ly-1109 : $11800 \pm 300$ BP).

7 La nature du gisement posait problème : habitat de plein air, vidanges répétées de la grotte des Vénus, mélange des deux ? C'est essentiellement pour tenter de répondre à ces questions qu'un nouveau sondage a été effectué par l'auteur en 1994. 


\section{Le sondage}

8 Le sondage principal, de $2 \mathrm{~m}$ par $1 \mathrm{~m}$, orienté nord-sud, situé à proximité immédiate du sondage de $\mathrm{H}$. Bessac, se trouve en contrebas de la grotte des Vénus. Fouillé jusqu'à une profondeur de $1,40 \mathrm{~m}$ sur $2 \mathrm{~m} 2$, il a été poursuivi par une fenêtre de $1 / 4$ de mètre carré jusqu'à 1,90 m sans que la base des niveaux d'occupation ait été atteinte. La fouille a été abandonnée à cette profondeur lorsqu'il n'a plus été matériellement possible de travailler. En raison de l'importante épaisseur des niveaux archéologiques, de l'abondance du matériel mis au jour, et de la difficulté d'interprétation du site, il a paru préférable d'arrêter là l'opération. Le site est ainsi préservé au maximum pour une étude ultérieure plus approfondie.

9 La stratigraphie est la suivante (fig. 1):

Figure 1 : Stratigraphie.

Figure 1 : Stratigraphy.

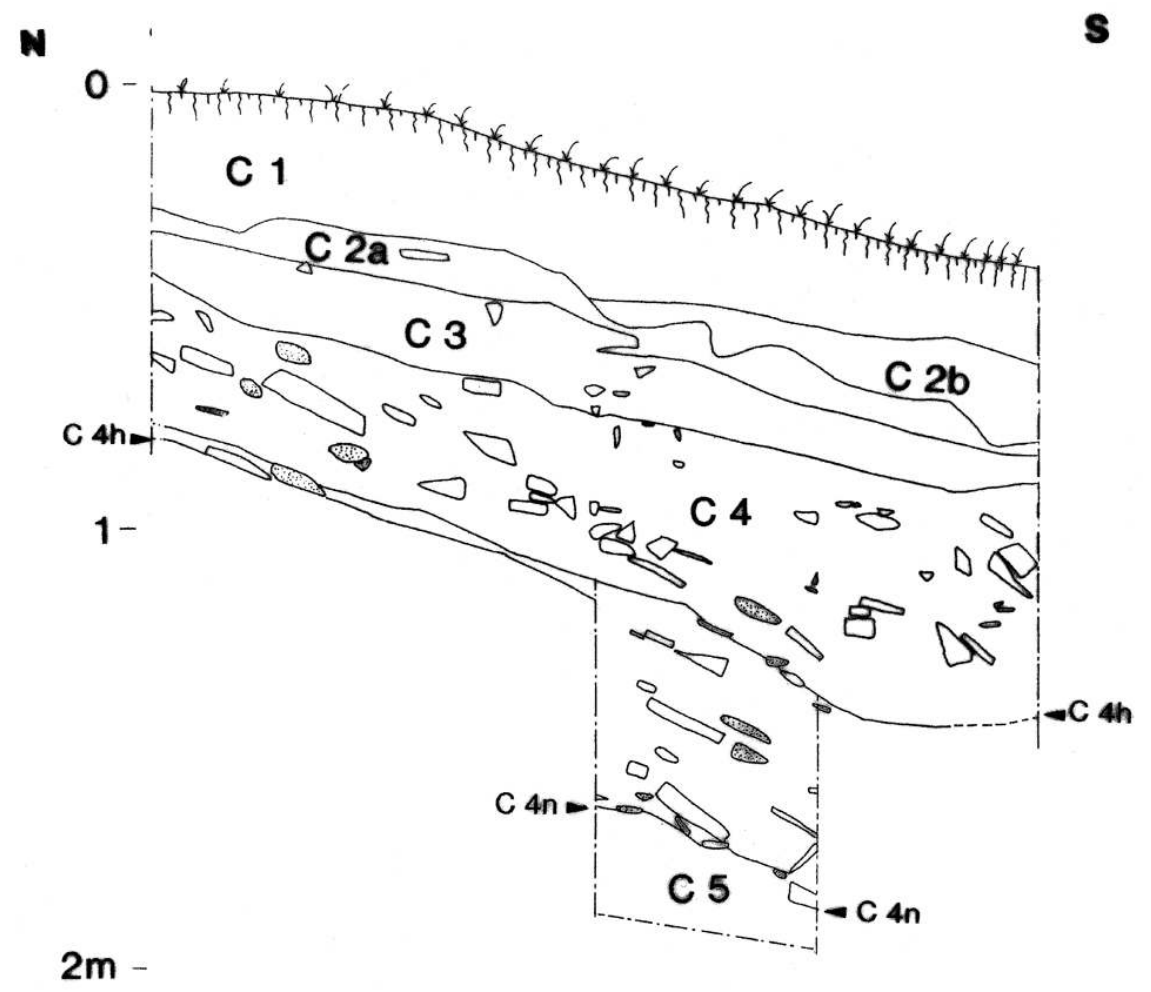

10 C $1: 0,40 \mathrm{~m}$ : humus

C 2 a : $0,05 \mathrm{~m}$ : argile rouge

C 2 b : 0,06 $\mathrm{m}$ : argile jaune

C $3: 0,10 \mathrm{~m}$ : cendres blanches

C $4: 1 \mathrm{~m}$ à $1,10 \mathrm{~m}$ : sédiment très noir, charbonneux, cailloux et blocs calcaires, galets de quartz, gneiss, schiste, interstratifié en 14 niveaux, ( $C 4 a$ à $C 4 g$ ) très riches en mobilier et faune. Pendage assez accentué (environ $12^{\circ}$ )

C 5 : puissance inconnue, fouillé sur $0,30 \mathrm{~m}$ : éboulis calcaire, sédiment interstitiel très noir, mobilier et faune abondants.

Dans le niveau C 4 g, on note, en partie engagé dans la coupe, un foyer en cuvette bordé de pierres, surmonté de gros blocs anguleux. 


\section{Le matériel de la C 4} fourni l'essentiel du mobilier ainsi que trois des six plaquettes gravées, dont celle qui porte les figures féminines schématiques. Voici les principales données concernant ce niveau :

Industrie lithique : très abondante (plus de 5200 artefacts) et très variée, elle comporte 548 outils. On note une nette prépondérance des burins $(12,2 \%)$ sur les grattoirs $(6,02 \%)$ qui sont en même proportion que les perçoirs. Les burins dièdres l'emportent sur les troncatures (Ibd : 7,48\%).L'indice de microlithes atteint 60,04\%, les lamelles à dos représentent plus de la moitié de l'outillage $(54,18 \%)$, les pièces à coche $(6,39 \%)$ et les denticulés $(4,01 \%)$ sont en nombre non négligeable.

Industrie osseuse : moins abondante, elle comporte quelques fragments d'aiguilles et de sagaies ainsi que deux harpons. L'un, relativement complet, bien que les barbelures manquent, est bilatéral (fig. 2), l'autre, incomplet, est sans doute du même type puisqu'il montre deux tubercules au niveau de l'embase. Une radiodatation effectuée sur un fragment de bois de renne provenant du même niveau que le harpon bilatéral a donné un âge de $13620 \pm 130$ BP (GifA 96345).

Figure 2 : Harpon bilatéral.

Figure 2 : Double-barbed harpoon.

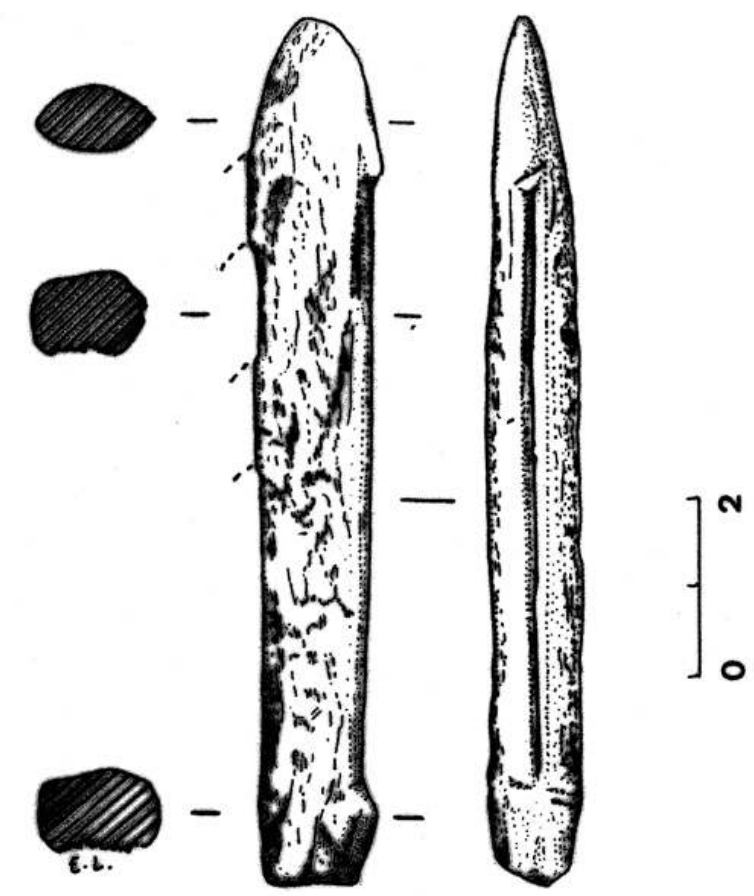

La présence de harpons permet d'attribuer l'industrie au Magdalénien supérieur.

16 probablement). On note la présence de cervidés (rennes et cerfs), de chevaux, de grands 
bovidés, de renards. Les restes d'oiseaux, comme les restes de poissons ne sont pas négligeables.

Restes humains: plusieurs dents humaines, trouvées mêlées à la faune, sont en cours d'étude.

Art mobilier: représenté par trois plaquettes de calcaire gravées, dont celle qui fait l'objet principal de ce travail. Trois autres plaquettes proviennent des déblais de $\mathrm{H}$. Bessac.

\section{Les plaquettes gravées}

19 A l'exception d'un exemplaire (fragment de stalagmite), ce sont des plaquettes en calcaire local, dont le poids varie de 1 à $11 \mathrm{~kg}$.

Nous distinguerons les plaquettes portant des décors figuratifs de celles qui en sont dépourvues, en commençant par ces dernières.

\section{A) Plaquettes à décor non figuratif}

\section{Plaquette A - Déblais Bessac (fig. 3)}

Figure 3 : Plaquette $A$ : relevé d'ensemble et coupe (relevé E.L.).

Figure 3: Plaquette A: general reading and section (reading E.L.).

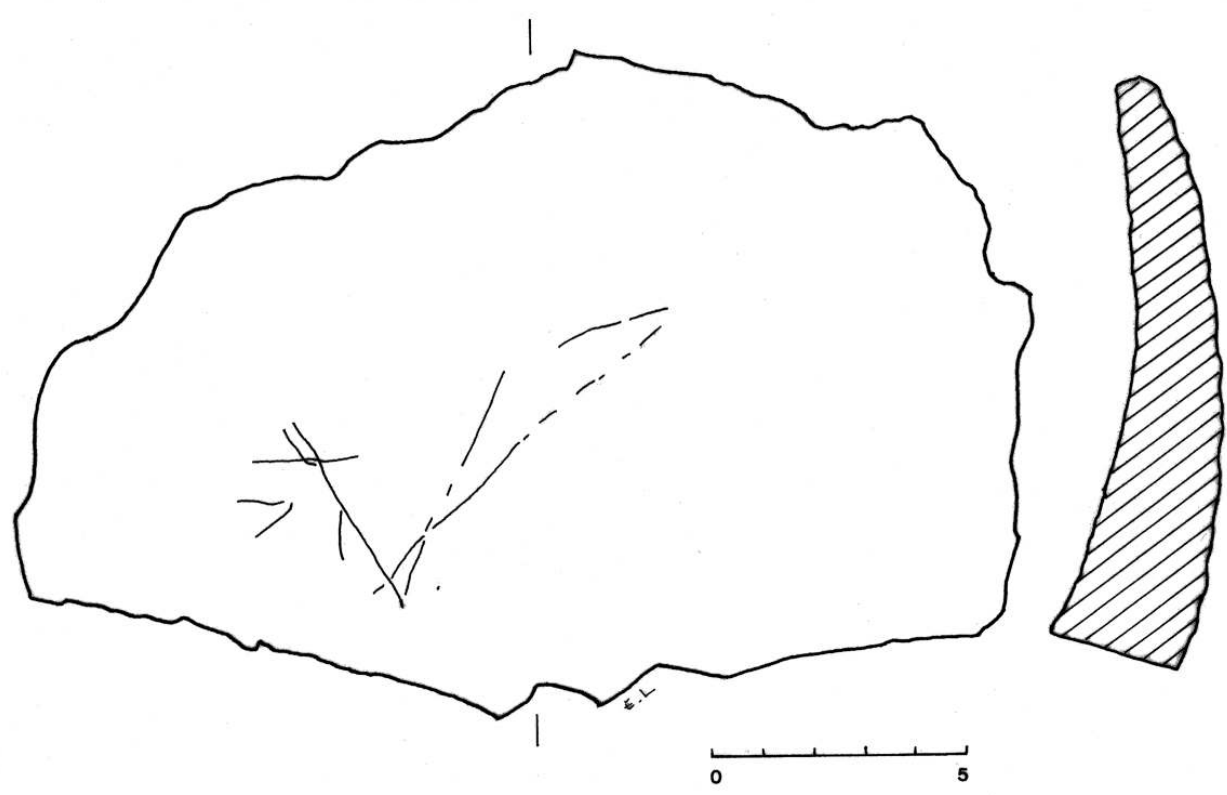




\section{Plaquette C - Déblais Bessac (fig. 4 et 5)}

Figure 4 : Plaquette $C$ : recto et coupe (relevé E.L.). Figure 4: Plaquette C: recto and section (reading E.L.).

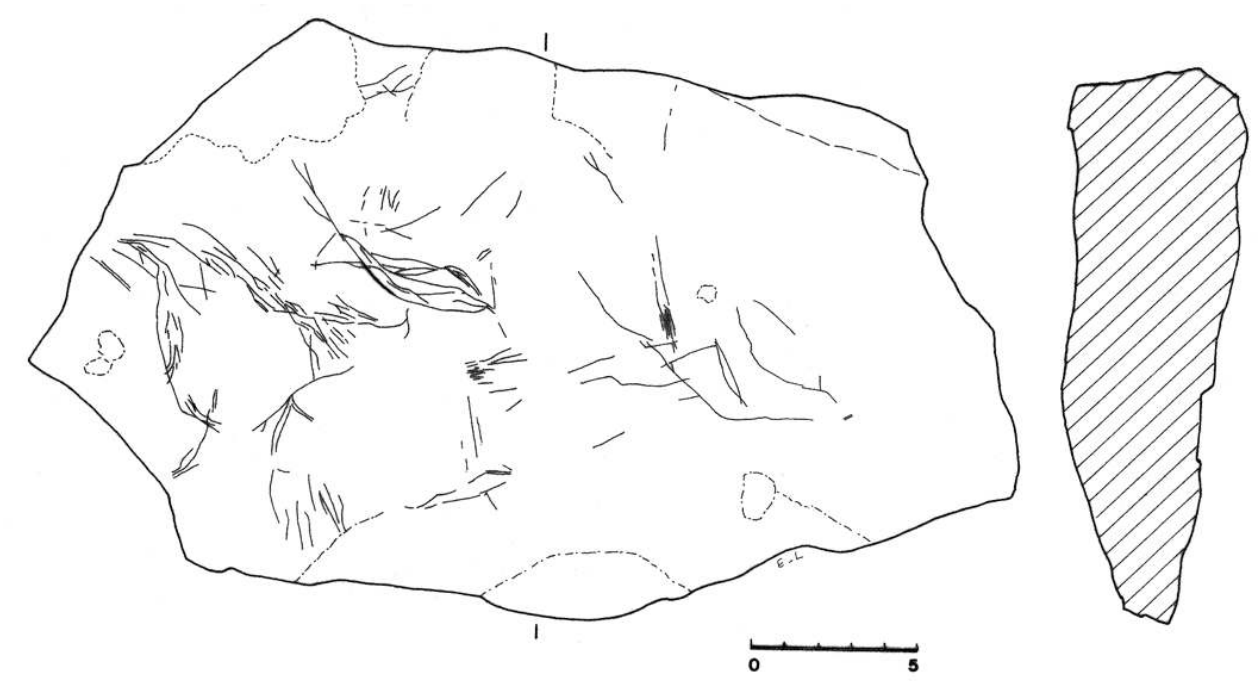

Figure 5 : Plaquette $C$ : verso (relevé E.L.).

Figure 5 : Plaquette $C$ : verso (reading E.L.).

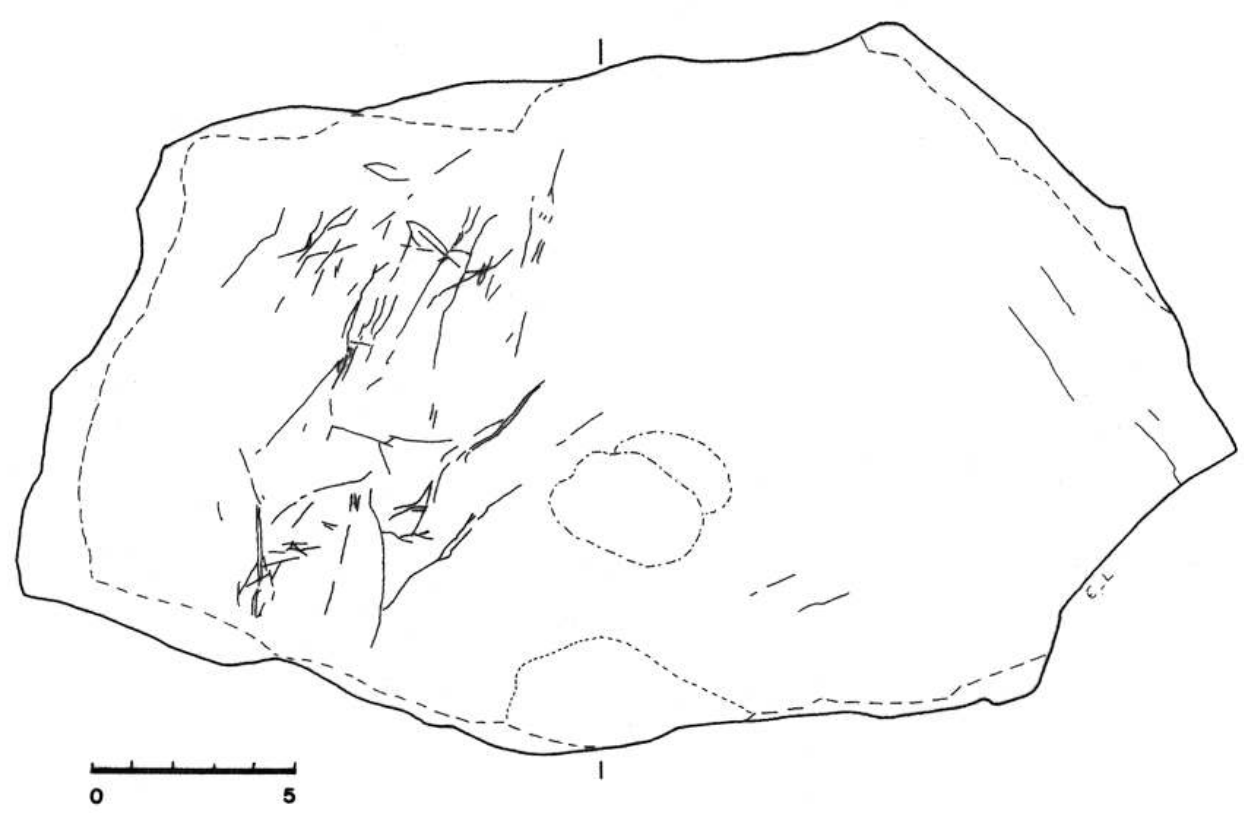

Longueur max. : 28,5 cm ; largeur max. : 17,5 cm ; épaisseur : de 3 à $3,5 \mathrm{~cm}$; poids : 3,8 $\mathrm{kg}$.

Plaquette de calcaire beige jaunâtre, de forme grossièrement hexagonale allongée, décorée sur les deux faces.

Recto: surface assez plane peu accidentée, portant vers le milieu une dépression naturelle ovale, aux bords bien délimités. Un second creux assez semblable se situe sur le bord inférieur. 
La partie gauche de la plaquette porte un décor non figuratif constitué d'une série de tracés fins, plus ou moins rectilignes ou courbes. Vers le bord gauche, un léger ressaut de la surface est souligné à la base par un étroit faisceau de traits fins. Quelques traits isolés occupent l'extrémité droite de la pièce (fig.4).

Verso : surface très accidentée, bosselée, portant des zones avec des concrétions de calcite. La partie droite était recouverte d'épaisses concrétions cendreuses, qui ont été enlevées à l'aiguille, sous loupe grossissement x 2 , afin de dégager les traits gravés sous-jacents. La presque totalité de la surface est recouverte d'une fine couche d'ocre ; les $2 / 3$ droits portent en outre des plages noirâtres, probablement brûlées. Le décor gravé sur la partie gauche de la plaquette est postérieur au dépôt d'ocre : les traits se détachent en beige clair sur le fond rosé. En revanche, dans la zone droite, le fond des traits les plus profonds est encombré d'un épais dépôt d'ocre : les gravures sont donc antérieures (fig. 5).

Plaquette F - C 4 b (fig. 6)

Figure 6 : Plaquette $\mathrm{F}$ : relevé d'ensemble et coupe (relevé E.L.). Figure 6: Plaquette F: general reading and section (reading E.L.).

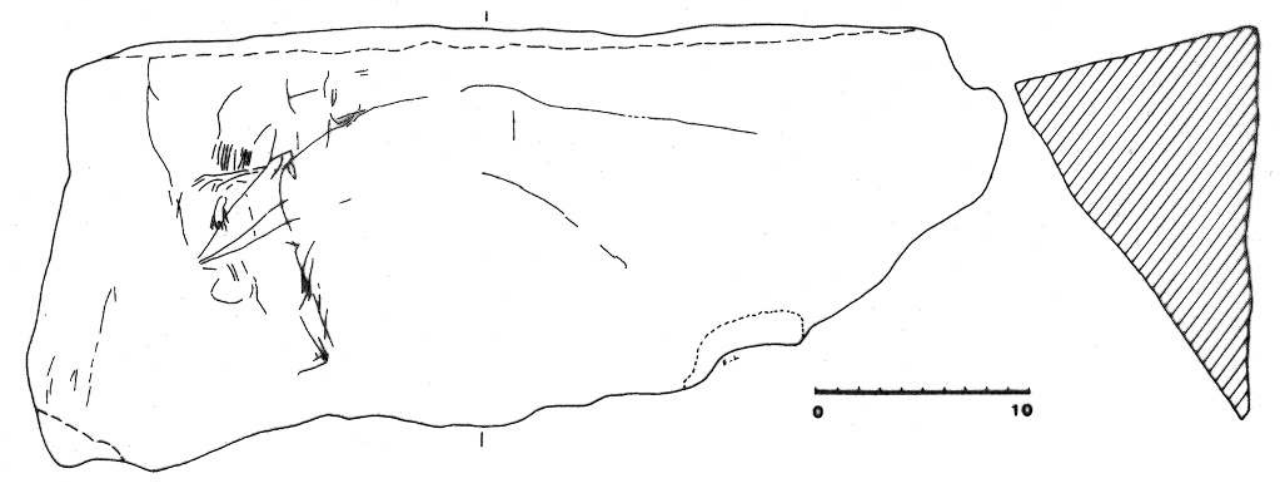

Longueur max. : 46,5 cm ; largeur max. : $19 \mathrm{~cm}$; épaisseur max. : 9,5 cm ; poids : $11 \mathrm{~kg}$.

Grande plaquette de forme grossièrement triangulaire, en calcaire beige clair, avec une patine rougeâtre, couverte d'épaisses concrétions grisâtres, cendreuses, qui ont été retirées à l'aiguille pour dégager le décor. Celui-ci n'existe que sur une face. La surface décorée affecte la forme générale d'un triangle allongé; elle est grenue mais pas trop irrégulière. Le décor se concentre vers le bord gauche de la plaquette, dans une concavité qui s'étend du bord supérieur au bord inférieur. Centré plutôt vers le bord supérieur, ce décor consiste en traits plus ou moins profonds, d'orientations diverses, qui ne montrent aucune représentation figurative.

\section{B) Plaquettes portant un décor figuratif}

\section{Plaquette B - Déblais Bessac (fig. 7 et 8)}


Figure 7 : Plaquette $B$ : relevé d'ensemble et coupe (relevé E.L.). Figure 7: Plaquette B: general reading and section (reading E.L.).

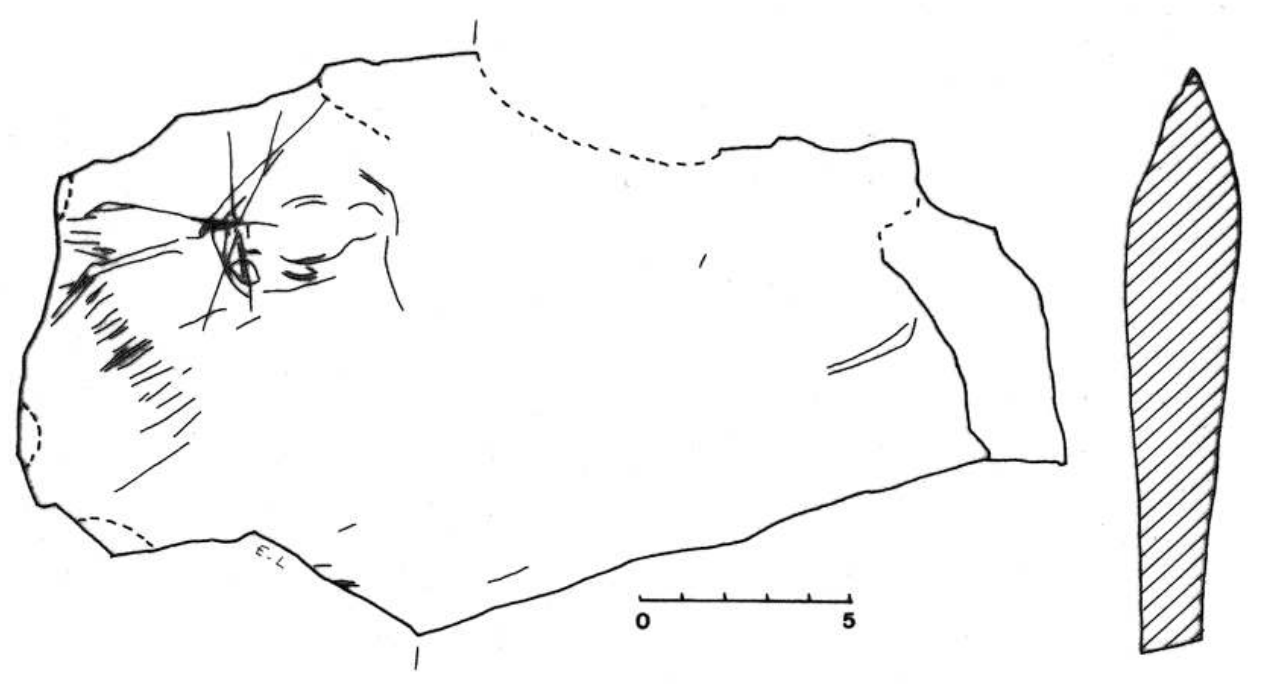

Figure 8 : Plaquette $B$ : relevé sélectif de la tête de bison (relevé E.L.). Figure 8: Plaquette B: selective reading of the bison head (reading E.L.).

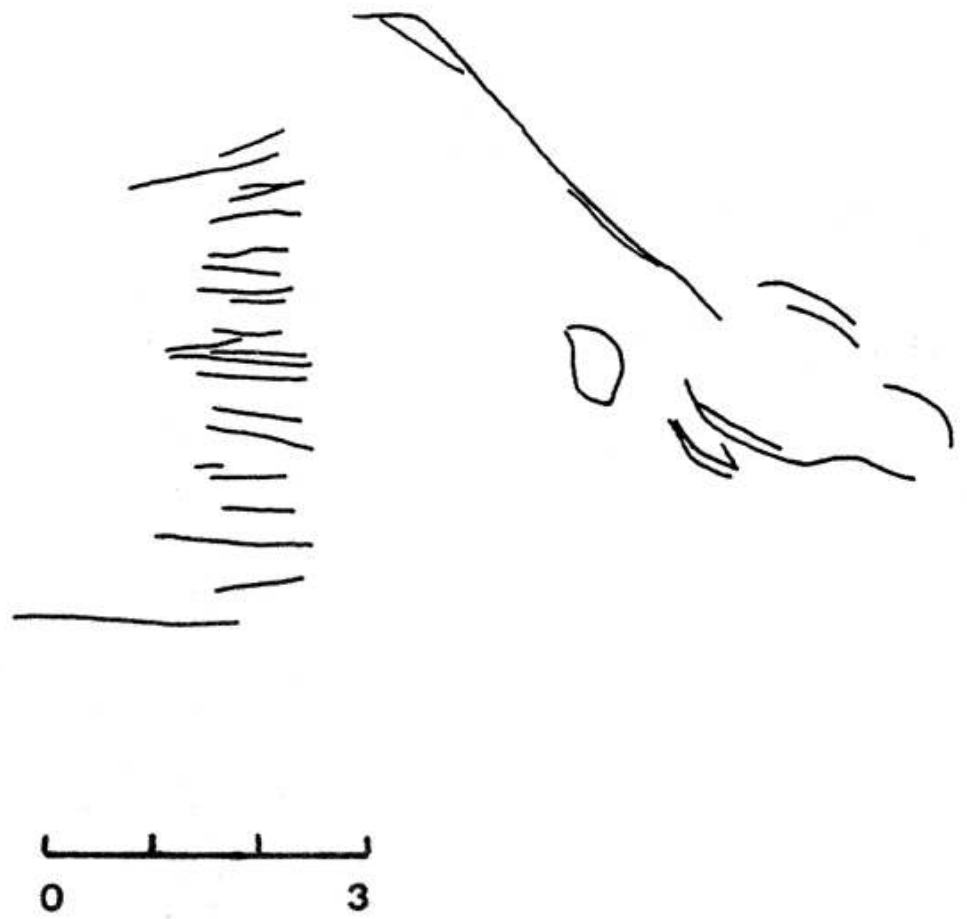

Longueur max. : 24,5 cm ; largeur max. : $14 \mathrm{~cm}$; épaisseur : de 0,5 à $3,5 \mathrm{~cm} ;$ poids : 1,350 $\mathrm{kg}$.

31 Calcaire beige clair, grossièrement en forme d'hexagone allongé. Le recto montre une surface assez lisse et régulière, portant une légère protubérance vers le haut, au milieu. Le verso est assez semblable ; il montre en outre des concrétions brun clair. 
Le recto porte un décor figuratif, centré vers le bord gauche de la plaquette.

1. une tête de bison, très simplifiée, tournée à gauche : cornes en accolade, de tracé discontinu, dirigées vers l'arrière, représentées en perspective, chanfrein, amorce du mufle, naseau possible, barbe et fanon traités en longues hachures parallèles. On distingue également une oreille, petite, ourlée, sous la corne gauche, et un œil, grand, bien dessiné par un trait large et peu profond. Le tracé du chanfrein, très accentué, repassé, souligne la base d'un léger ressaut de la surface. Le tracé est discontinu, l'assemblage des divers éléments de cette tête étant visuel (fig. 7 et 8).

2. un faisceau de traits plus ou moins profonds, presque raclés pour certains, postérieur au chanfrein qu'il recoupe. Quatre traits parallèles, dans le prolongement de la barbe, à l'emplacement du mufle absent, sont antérieurs au tracé du chanfrein qui en recoupe un. La partie droite de la plaquette porte deux tracés légèrement courbes et subparallèles.

\section{Plaquette D - C 4 (fig. 9 et 10)}

Figure 9 : Plaquette $D$ : relevé d'ensemble et coupe (relevé E.L.). Figure 9: Plaquette D: general reading and section (reading E.L.).

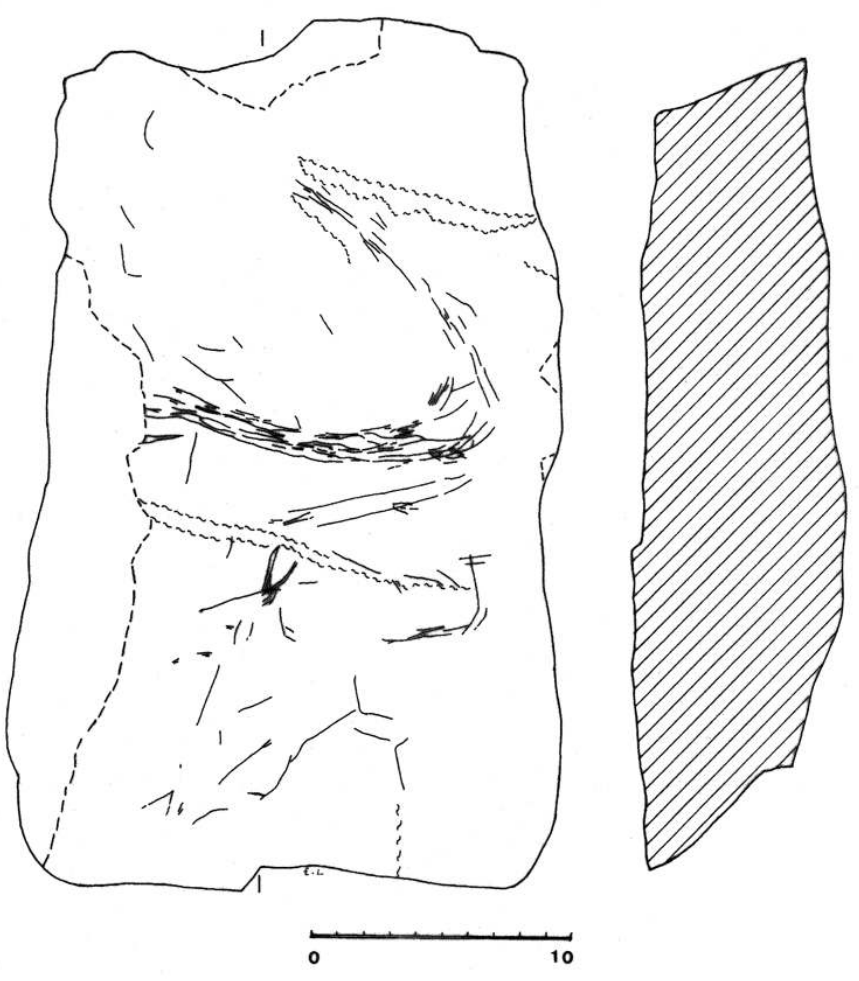


Figure 10 : Plaquette $D$ : a : Relevé sélectif de la tête de daguet (relevé E.L.) ; b : Daguet, d'après Grzimek et Fontaine, 1972, t. 13, p. 66.

Figure 10: Plaquette D: a: selective reading of young deer head (reading E. L.); b: Young red deer, after Grzimek and Fontaine, 1972, t. 13, p. 66.
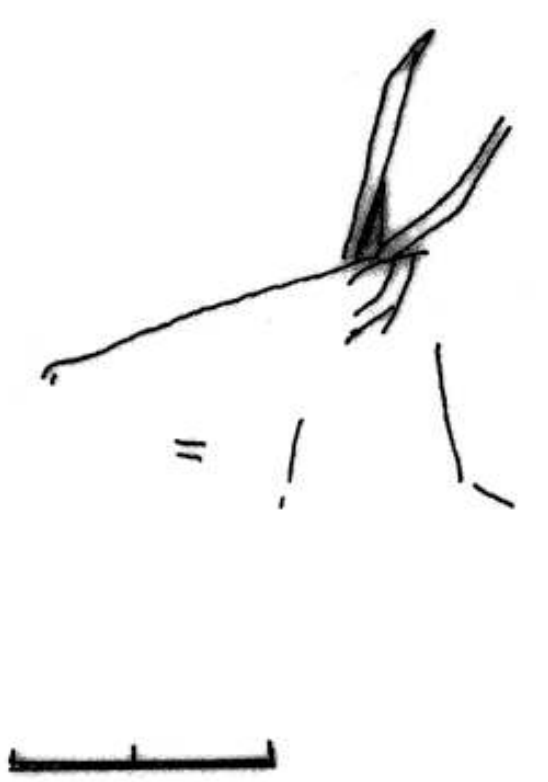

0 central, se distingue une petite tête animale tournée à gauche, en position médiane. Esquissée, incomplète, elle comporte un chanfrein avec l'amorce du mufle et une oreille ourlée bien dessinée, surmontée de deux “ cornes " étroites, en croissant. Ni les yeux, ni les naseaux, ni la bouche ne sont figurés. L'encolure est esquissée. La minceur des cornes légèrement indiquées par deux traits, comme leur courbure divergente, exclut la représentation d'un bouquetin ou d'un chamois (fig. 10a et 10b). Les tracés sont fins mais vigoureux.

Cette tête, d'allure gracile, portée droite sur l'encolure, peut être interprétée comme la représentation d'un daguet, cerf de un an à un an et demi, dont la ramure se limite à une perche non ramifiée (Grzimek et Fontaine 1972). 
Plaquette E - C 4 (fig. 11 et 12)

Figure 11 : Plaquette $\mathrm{E}$ : relevé d'ensemble et coupe (relevé E.L.).

Figure 11: Plaquette E: general reading and section (reading E.L.).

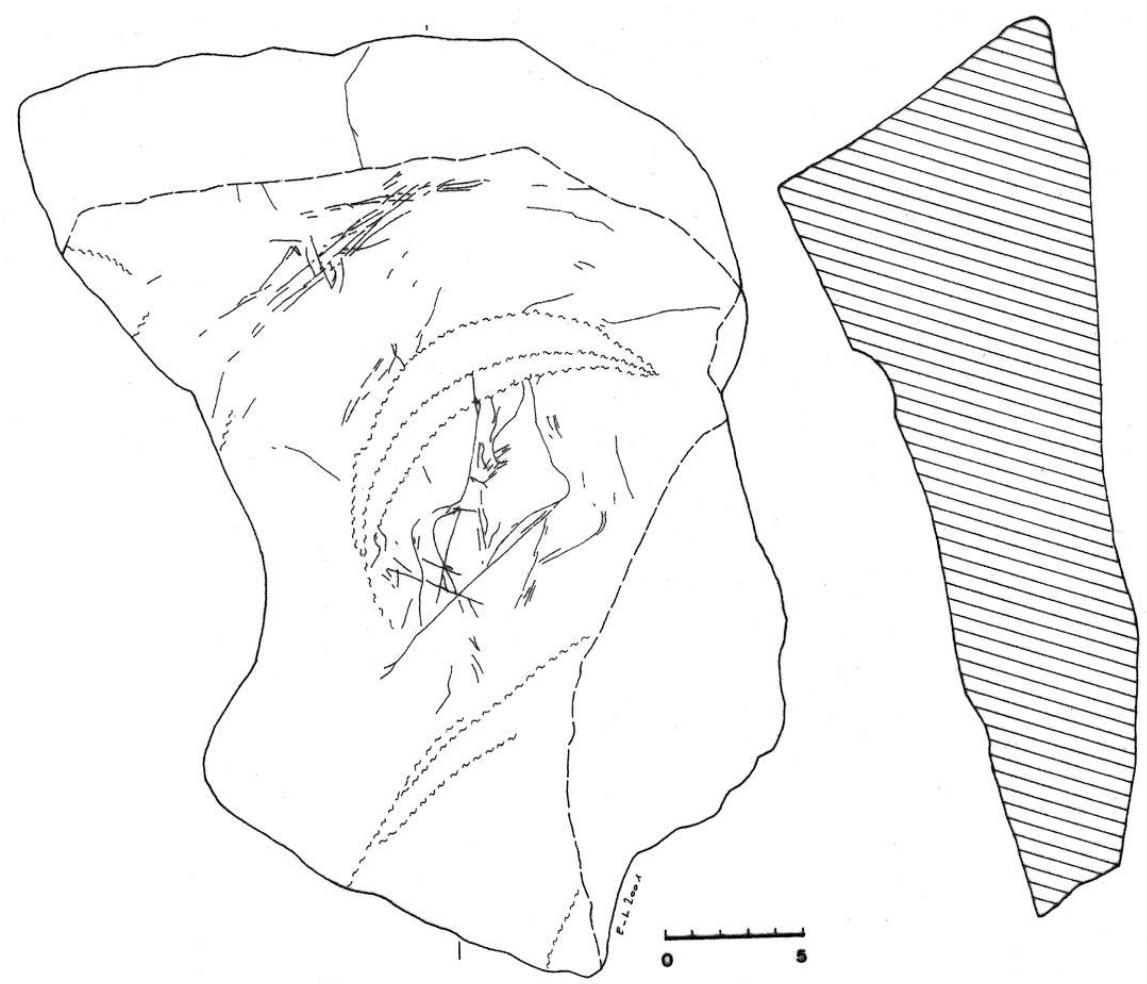


Figure 12 : Plaquette $\mathrm{E}$ Figure 12: Plaquette $E$

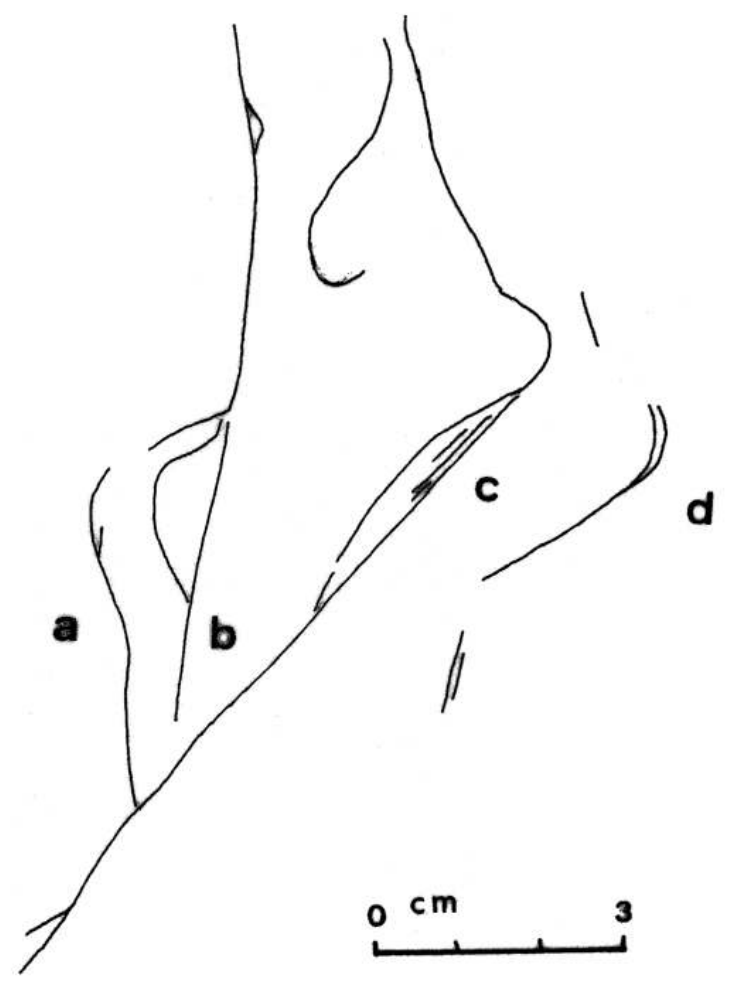

a et $b$ : figures féminines schématiques tournées à droite ; $c$ et $d$ : figures féminines schématiques tournées à gauche (relevé E.L.).

$a$ and b: right oriented female figures; $c$ and d: left oriented female figures (reading E.L.). 
47 Figure $b$ : tournée à droite, emboîtée dans la précédente : ligne de dos esquissée, fessier proéminent, pas de pliure du genou. Utilise peut-être la ligne de ventre de la précédente.

Ces deux figures sont gravées légèrement, au moyen de traits dissymétriques en $\mathrm{V}$, fins, usés.

Figure $c$ : tournée à gauche : ligne dorsale prenant naissance dans le bord du godet, fessier peu volumineux mais bien marqué, ligne postérieure de la jambe légèrement concave, prolongée par un long tracé rectiligne. Le cou est indiqué, son tracé antérieur se poursuit par une ligne courbe, continue, qui paraît représenter un sein de profil, volumineux. Il serait disproportionné avec le fessier, selon les canons habituels de ce type de figuration, où les seins sont absents ou menus (Bosinski et Fischer 1974).

50 Figure d: tournée à gauche : longue ligne de dos, arrondi du fessier, ligne postérieure de la cuisse dans le prolongement de celui-ci, indication possible de la pliure du genou (fig. 12).

51 Le décor de la plaquette paraît constituer une composition, organisée autour du " godet" central : deux groupes de traits paraissant encadrer celui-ci en suivant son contour, deux groupes de deux femmes centrées à l'intérieur. L'élément principal de la composition est le groupe de quatre femmes, emboîtées deux à deux et disposées face à face.

52 Divers éléments montrent l'existence de relations entre les deux groupes de deux femmes : leur situation dans le "godet", leur disposition en face à face, les différences morphologiques entre les deux groupes. Celles orientées à droite sont sveltes, élancées, représentées debout; celles orientées à gauche paraissent plus massives et sont représentées en position assise. Cette différence qui marque une opposition entre les deux groupes indique-t-elle une scène? Il est difficile de l'affirmer. En tout état de cause, cette opposition renforce encore l'unité de la composition de ce groupe de femmes.

Les représentations féminines schématiques mobilières, gravures ou statuettes, ne sont pas rares dans la vallée de l'Aveyron: on dénombre neuf gravures et deux statuettes à l'abri de Fontalès, une statuette et deux gravures à la grotte du Courbet (Ladier 1992, Welté et Ladier 1995). Ces quatre nouvelles figures féminines schématiques de type Lalinde-Gönnersdorf complètent les inventaires de ce type de figurations, montrant dans la vallée de l'Aveyron la principale concentration des figures féminines schématiques mobilières du Quercy (Ladier, Lenoir, Welté sous presse).

\section{Conclusion}

Le site magdalénien supérieur de la Magdeleine-La Plaine, exploré sur une surface très restreinte, se caractérise par l'abondance de son industrie lithique et la présence d'art mobilier sur support lithique, en particulier de figures féminines.

superficie restreinte du sondage n'a pas permis de statuer sur la nature exacte du remplissage anthropique, d'autant que celui-ci n'a pas pu être exploré sur toute son épaisseur. Plusieurs hypothèses peuvent être envisagées.

L'existence d'une stratification nette et d'un foyer dans la C 4 semblent indiquer un habitat de plein air. La présence de figures féminines schématiques paraît constituer un 
argument supplémentaire dans ce sens, car dans la vallée de l'Aveyron toutes les autres figures de ce type proviennent d'habitats (Fontalès, le Courbet). Pourtant, cette interprétation peut être infirmée par le pendage du niveau, environ $12^{\circ}$, qui paraît incompatible avec un habitat, bien que nombre d'habitats de plein air paléolithiques occupant des terrasses situées au-dessus de cours d'eau montrent une pente sensible. C'est le cas par exemple à Gönnersdorf, où cette particularité pouvait permettre une bonne évacuation des eaux pluviales, et contribuer ainsi à la salubrité des habitations (G. Bosinski, communication personnelle). Compte tenu de la faible superficie explorée, le doute subsiste sur l'existence d'un habitat, sans que cette hypothèse puisse être totalement écartée.

La situation du gisement, en contrebas de la grotte ornée, peut permettre de penser que le dépôt archéologique s'est constitué par des vidanges successives de la cavité, effectuées au Paléolithique. La stratification observée pourrait résulter de cette opération. La grotte a été vidée au siècle dernier, mais des restes du remplissage soudés aux parois par de la calcite contiennent encore du matériel archéologique. F. Rouzaud évoque un "dégagement périodique de la base des sculptures" (Rouzaud et alii, op. cit.) par les paléolithiques, visant à ce qu'elles ne soient pas recouvertes par le remplissage. Mais rien ne prouve qu'ils évacuaient le sédiment. Le volume des niveaux anthropiques, l'étendue du gisement, et surtout sa large extension de part et d'autre de la grotte constituent autant d'obstacles à cette interprétation. En effet, le faible volume de cette cavité aurait nécessité des vidanges répétées, sur une très longue période, que rien ne peut démontrer. Là encore, nous n'avons que trop peu d'éléments pour trancher.

On peut envisager enfin que la constitution des niveaux archéologiques soit due à l'accumulation ou à la succession des deux causes précédentes.

La nature du gisement reste donc hypothétique, plusieurs possibilités étant envisageables. En tout état de cause, la présence des figures féminines schématiques permet de rattacher le site, quelle que soit sa nature, au groupe culturel qui occupait les sites de Fontalès et du Courbet (Ladier et Welté sous presse).

L'existence d'une relation entre le gisement de plein air et la grotte ornée, si elle paraît probable, reste à établir et éventuellement à déterminer dans sa nature. Il en va de même en ce qui concerne d'éventuelles relations entre les femmes mobilières et les femmes pariétales. Ces questions sont particulièrement épineuses, puisque les œuvres de la grotte sont attribuées au Magdalénien moyen au plus tard, alors que le gisement se rapporte au Magdalénien supérieur.

\section{BIBLIOGRAPHIE}

BESSAC, H. 1971. L'ensemble aux Vénus gravées de la grotte de la Madeleine de Penne. Revue du Tarn, 1971, 63, p. 325-334.

BESSAC, H. 1977. Magdalénien final de plein air à la Madeleine de Penne (Tarn). Bulletin de la Fédération Tarnaise de Spéléo-Archéologie, Travaux et Recherches n 14, 1977, p. 87-11. 
BOSINSKI, G., FISCHER, G. 1974. Die Menschendarstellungen von Gönnerdorf :Ausgrabung von 1968.Wiesbaden : Franz Steiner Verlag, GMBH.

BREUIL, H. 1952. Les bas-reliefs de la Magdeleine à Penne (Tarn). Comptes rendus de l'Académie des Inscriptions et Belles Lettres, 1952, p. 612-614.

GRZIMEK, B., FONTAINE, M. 1972. Le Monde animal en 13 volumes. Zurich : Stauffacher, 1972. Tome XIII, p. 166.

LADIER, E. 1992. La Vénus du Courbet. L'Anthropologie, 1992, 96, 2-3, p. 349-356.

LADIER, E. 1995. Penne, La Magdeleine-La Plaine. SRA Midi-Pyrénées : Bilan scientifique 1994, p. 204.

LADIER, E. 1996. Le gisement magdalénien de plein air de La Magdeleine-La Plaine (81 206002), Tarn. Rapport de sondage 1994-1995, Musée d'histoire naturelle de Montauban-SRA Midi-Pyrénées, 1996, 16 p., annexes, 22 pl., photos.

LADIER, E., LENOIR, M., WELTÉ, A.-C. (sous presse). Relations ou convergences entre Périgord et Quercy : le cas des Figures Féminines Schématiques de type Lalinde-Gönnersdorf. In : Terres et Hommes du Sud, $26^{\circ}$ Congrès du CTHS, Toulouse, 2001.

LADIER, E., WELTÉ, A.-C. (sous presse). Territoires culturels au Magdalénien supérieur dans la vallée de l'Aveyron : éléments d'approche. In : Terres et Hommes du Sud, $26^{\circ}$ Congrès du CTHS, Toulouse, 2001.

ROUZAUD, F., BISIO, A., LAUTIER, J., SOULIER, M. 1989. Grotte de La Magdeleine-des-Albis, à Penne (Tarn).Essai de synthèse à l'occasion de la découverte d'une nouvelle figure féminine. Préhistoire Ariégeoise, 1989, 44, p. 21-61.

TISNERAT-LABORDE N., VALLADAS, H., LADIER, E. 1997. Nouvelles datations carbone 14 en SMA pour le Magdalénien supérieur de la vallée de l'Aveyron. Préhistoire Ariégeoise, 1997, 52, p. 129-135. WELTÉ, A.-C., LADIER, E. 1995. Les figures féminines des sites magdaléniens de la vallée de l'Aveyron. In : La Dame de Brassempouy. Colloque international de Brassempouy (juillet 1994). Liège : ERAUL 74, 1995, p. 273-284.

\section{RÉSUMÉS}

Lors d'un sondage réalisé en 1994 sur le site magdalénien supérieur de La Magdeleine-La Plaine, plusieurs plaquettes gravées ont été mises au jour. L'une d'elles porte quatre figures féminines schématiques de type Lalinde-Gönnersdorf.

On the occasion of a small excavation in the Upper Magdalenian site of La Magdeleine-La Plaine (Penne, Tarn, France), several stone engraved plaquettes were discovered. One of them presents four schematic female figures related to the type of Lalinde-Gönnersdorf.

\section{INDEX}

Mots-clés : figures féminines schématiques, Magdalénien supérieur, vallée de l'Aveyron

Keywords : Aveyron valley, schematic female figures, Upper Magdalenian 
AUTEUR

EDMÉE LADIER

Conservateur en Chef / Musée d'histoire naturelle - 2, place Antoine Bourdelle 82000 Montauban 